\section{E-181 RADIOLOGIC AND CLINICAL OUTCOME OF COIL EMBOLIZATION FOR RESIDUAL ANEURYSM AFTER SURGICAL CLIPPING}

J Baek*, H Jeong. Diagnostic Radiology, Inje University Busan Paik Hospital, Busan, Korea, Republic of

\subsection{6/neurintsurg-2020-SNIS.212}

Introduction and Purpose Because postoperative adhesion and re-craniotomy have been limitations of surgical revision in the residual aneurysms after clipping, so coil embolization has been used as an alternative technique for surgical clipping in the residual aneurysm after clipping. In this study, we investigated the clinical and radiological results of patients treated with coil embolization for residual aneurysms after surgical clipping retrospectively.

Materials and Methods Between 1999 and 2018, 19 aneurysms of 19 patients who underwent coil embolization for residual aneurysms observed after clipping or wrapping were studied.

Results Eight patients presented with subarachnoid hemorrhage at the time of clipping (42.1\%). The median interval between surgical clipping and coil embolization was 21 days (range, 02595 days). Techniques of coil embolization included single catheter $(n=15)$, stent assisted $(n=3)$, and stent deployment only without coiling $(n=1)$. Immediate radiologic findings after coil embolization showed complete occlusion in 5 patients, a residual neck in 10 patients, and a residual sac in 4 patients. There was no procedural morbidity and mortality. Intraprocedural rupture was occurred in one case, but there was no neurological deficits after procedure. All patients of residual aneurysm after surgical clipping treated by coil embolization were available for clinical follow-up. The mean clinical follow-up was $33.4 \pm 24.7$ months. Poor clinical outcomes (modified Rankin Scale score $\geq 3$ ) at the end of the clinical follow-up were reported in 4 patients (21\%). However, all four patients had the same clinical results as before the coil embolization. Angiographic follow-up was available for 14 patients (73.7\%). Minor recanalization was detected in only 1 patient, but no need to re-treatment (7.1\%).

Conclusions According to our study, coil embolization for residual aneurysm after surgical clipping may probably be a feasible, safe, and relative durable treatment modality.

Disclosures J. Baek: None. H. Jeong: None.

\section{E-182 THE PRIMARY EXPERIENCE OF USING A COMANECI EMBOLIZATION ASSIST DEVICE DURING EMBOLIZATION OF INTRACRANIAL ANEURYSMS WITH DETACHABLE MICROCOILS}

K Orlov*, N Strelnikov, V Berestov. Meshalkin National Medical Research Center Novosibirsk, Russian Federation

\subsection{6/neurintsurg-2020-SNIS.213}

Background Endovascular treatment of intracranial aneurysms using detachable microcoils is among the most effective methods for excluding aneurysms from the circulation. Auxiliary procedures allow one to significantly increase the radicality of embolization, especially for wide-necked aneurysms.

Objective To evaluate the effectiveness and safety of the Comaneci device in endovascular treatment of intracranial aneurysms using microcoils.

Materials and Methods Twelve unruptured intracranial aneurysms in 10 patients were embolized with the Comaneci device at the National Medical Research Center named after Academician E.A. Meshalkin in October-December, 2019 (including two (16.7\%) AVM-associated proximal flow-related aneurysms in one patient). All the aneurysms were located in the carotid basin; the dome-to-neck ratio was $<2$.

Results The technical success of embolization was achieved in all the cases. Total embolization (class I according to the Raymond-Roy Occlusion Classification) was observed in 11 (91.7\%) cases. One (8.3\%) patient had incomplete occlusion (class IIIb according to the Raymond-Roy Occlusion Classification). In one $(8.3 \%)$ case, a single coil turn prolapsed into the mother vessel lumen when the device was removed, so a stent had to be inserted. The disability and mortality rates were $0 \%$.

Conclusions Our primary experience of using the Comaneci device demonstrates that it allows one to achieve good angiographic and clinical outcomes during endovascular treatment of wide-necked intracranial aneurysms, thus being a good alternative to balloon- and stent-assisted coiling procedures. However, the study needs to be continued to evaluate safety of this device and the long-term outcomes of treatment.

Disclosures K. Orlov: None. N. Strelnikov: None. V. Berestov: None.

\section{E-183 FLOW DIVERSION VS COILING IN SMALL AND MEDIUM- SIZED ANEURYSMS OF THE SUPERIOR HYPOPHYSEAL ARTERY}

A Gorbatykh*, K Orlov. Meshalkin National Medical Research Center, Novosibirsk, Russian Federation

\subsection{6/neurintsurg-2020-SNIS.214}

Background Small and medium-sized aneurysms of the superior hypophyseal artery (SHA) comprise one-third of supraclinoid carotid lesions and are known for good results of endovascular treatment. Most of them are suitable for both flow diversion (FD) and coiling. The aim of this study was to compare the angiographic and clinical outcomes of both methods. The hypothesis was that FD provides better occlusion rates with similar procedural risks.

Materials and Methods Seventy-five SHA aneurysms sized between 4 and $12 \mathrm{~mm}$ were consecutively treated in a single center between 2014 and 2019. We retrospectively evaluated digital subtraction angiography (DSA) series and patient records, and recorded the demographics, aneurysm morphology, angiographic, and clinical outcomes. Total occlusion was defined as no filling at all. 'Acceptable' occlusion was defined as total OR subtotal occlusion, which remains stable during follow-up, with no signs of recanalization and no need for retreatment or further observation.

Results Twenty-six aneurysms were flow-diverted (36.4\%); 49 aneurysms were coiled (65.4\%). There was no difference between the groups in terms of aneurysm size (FD: median 7 mm, IQR 4; Coils: median $6 \mathrm{~mm}$, IQR 3; p=0.51), neck size (FD: $4 \mathrm{~mm}$, IQR 2; Coils: $5 \mathrm{~mm}$, IQR 2, $\mathrm{p}=0.51$ ), age $(p=0.65)$, sex $(p=0.57)$, and rate of symptomatic lesions $(p=0.49)$. Follow-up DSA was available for all FD and 49/53 coiled lesions (92.4\%).Definitive angiographic cure was achieved in 23/26 flow diverted cases (88.4\%) and 28/49 coiled cases $(57.1 \%) ; p=0.008$. The rate of clinically acceptable occlusion was $24 / 26$ in the FD group (92.3\%) and $43 / 49$ in the coil group $(87.7 \%) ; p=0.7$. There was one treatment- 